\title{
Nutritional labelling for promoting healthier food purchasing and consumption (Protocol)
}

\author{
Crockett RA, Hollands GJ, Jebb SA, Marteau TM
}

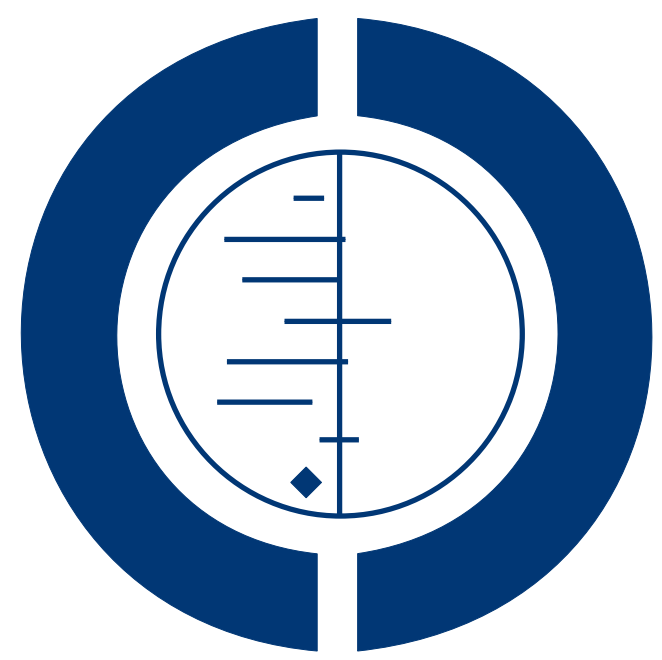

\section{THE COCHRANE COLLABORATION ${ }^{\circledR}$}

This is a reprint of a Cochrane protocol, prepared and maintained by The Cochrane Collaboration and published in The Cochrane Library 2011, Issue 9

http://www.thecochranelibrary.com

\section{WILEY}


TABLE OF CONTENTS

HEADER . . . . . . . . . . . . . . . . . . . . . . . . . . . . . . . . . . . . 1

ABSTRACT . . . . . . . . . . . . . . . . . . . . . . . . . . . . . . . . . . . . . .

BACKGROUND . . . . . . . . . . . . . . . . . . . . . . . . . . . . . . . . . . . . . . . . . . . . . .

Figure 1. . . . . . . . . . . . . . . . . . . . . . . . . . . . . . . . . . . . . .

Figure 2. . . . . . . . . . . . . . . . . . . . . . . . . . . . . . . . . . . . . . 44

OBJECTIVES . . . . . . . . . . . . . . . . . . . . . . . . . . . . . . . . . . . . . . . . . .

METHODS . . . . . . . . . . . . . . . . . . . . . . . . . . . . . . . . . . . . . . 6

ACKNOWLEDGEMENTS . . . . . . . . . . . . . . . . . . . . . . . . . . . . . . . . . 11

REFERENCES . . . . . . . . . . . . . . . . . . . . . . . . . . . . . . . . . . . . . 12

APPENDICES . . . . . . . . . . . . . . . . . . . . . . . . . . . . . . . . . . . . . 13

HISTORY . . . . . . . . . . . . . . . . . . . . . . . . . . . . . . . . . . . . . . . 15

CONTRIBUTIONS OF AUTHORS . . . . . . . . . . . . . . . . . . . . . . . . . . . . . . . . . . .

DECLARATIONS OF INTEREST . . . . . . . . . . . . . . . . . . . . . . . . . . . . . . . . .

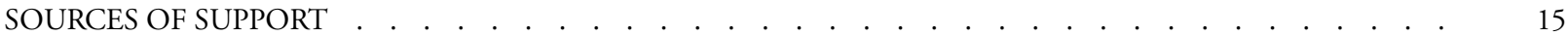

Nutritional labelling for promoting healthier food purchasing and consumption (Protocol)

Copyright (๑ 201 I The Cochrane Collaboration. Published by John Wiley \& Sons, Ltd. 


\title{
[Intervention Protocol]
}

\section{Nutritional labelling for promoting healthier food purchasing and consumption}

\author{
Rachel A Crockett ${ }^{1}$, Gareth J Hollands ${ }^{2}$, Susan A Jebb ${ }^{3}$, Theresa M Marteau ${ }^{1,2}$ \\ ${ }^{1}$ Health Psychology Section, King's College London, London, UK. ${ }^{2}$ Behaviour and Health Research Unit, Institute of Public Health, \\ University of Cambridge, Cambridge, UK. ${ }^{3}$ MRC Human Nutrition Research Unit, Medical Research Council, Cambridge, UK \\ Contact address: Rachel A Crockett, Health Psychology Section, King’s College London, 5th Floor Bermondsey Wing, Guy’s Campus, \\ London Bridge, London, SE1 9RT, UK. rachel.crockett@kcl.ac.uk.
}

Editorial group: Cochrane Public Health Group.

Publication status and date: New, published in Issue 9, 2011.

Citation: Crockett RA, Hollands GJ, Jebb SA, Marteau TM. Nutritional labelling for promoting healthier food purchasing and consumption. Cochrane Database of Systematic Reviews 2011, Issue 9. Art. No.: CD009315. DOI: 10.1002/14651858.CD009315.

Copyright (C) 2011 The Cochrane Collaboration. Published by John Wiley \& Sons, Ltd.

\begin{abstract}
A B S T R A C T
This is the protocol for a review and there is no abstract. The objectives are as follows:

The primary objective of this review is to assess whether nutritional labelling of foods in comparison to the same foods presented either
\end{abstract} without a label or with an incomplete label promotes:

1. healthier food purchasing behaviour from: a) restaurants, b) food shops, c) vending machines; or

2. healthier food consumption behaviour.

The secondary objective of the review is to explore possible modifiers of the effect of nutritional labelling on purchasing and consumption (described in more detail under 'Types of outcome').

\section{B A C K G R O U N D}

\section{Description of the condition}

Unhealthy eating contributes to increased prevalence of preventable diseases including obesity, cardiovascular disease, diabetes and many forms of cancer. In the United Kingdom (UK) alone it is estimated that 70,000 premature deaths could be avoided each year if eating behaviour matched nutritional guidelines (Cabinet Office 2008). These diseases pose a substantial threat to the health of populations and there is increasing concern about the challenges that they will pose to the effective and economic provision of health services (Finkelstein 2003; Foresight 2007). However, changing behaviour to reverse rises in potentially preventable disease is difficult. While many people want to engage in behaviours that promote good health, including healthy eating, people find it difficult to implement and maintain such behaviours (Ogden 2007; Sutton 1998). Eating healthily is made more difficult by an environment in which a great variety of tempting, convenient and cheap ready-to-eat foods are readily available and made highly salient through marketing. These foods are often energy dense (that is high in calories relative to their volume) and highly processed. They are packaged and marketed in ways that fail to help consumers understand their nutritional content. 


\section{Description of the intervention}

Until recently most food that was eaten was prepared and cooked at home from raw ingredients, making the contents apparent to those planning and preparing a household's meals. Progressively, however, fewer meals are being prepared from scratch and preprepared, often pre-packaged, meals are more and more forming a substantial part of dietary intake. These meals are often complex, consisting of a wide range of ingredients not all of which are familiar. Thus it can be difficult for those purchasing and consuming these meals to understand their nutritional content. Ingredient panels began to be provided to aid understanding of the nutritional content of pre-packaged foods. For example, in 1967, the USA Federal Trade Commission first enacted the Fair Packaging and Labelling Act requiring that food should be labelled to list its contents. In 1990 further legislation in the USA made some form of nutritional labelling compulsory on all pre-packaged foods. In the same year the European Union passed a directive to guide nutritional labelling, albeit voluntary (Campos 2011; Cowburn 2005). More sophisticated labelling of products has subsequently evolved to try to help people understand the nutritional composition of a product and how it might fit into a healthy diet. For example, in the UK two systems of nutritional labelling have been widely implemented.

1. Guideline daily amounts (GDA) labels which indicate the content of key nutrients contained in a portion as a percentage of the guideline daily amount (see Figure 1a).

Figure I. Example of nutritional labels

Figure 1a: Guideline Daily Amounts label

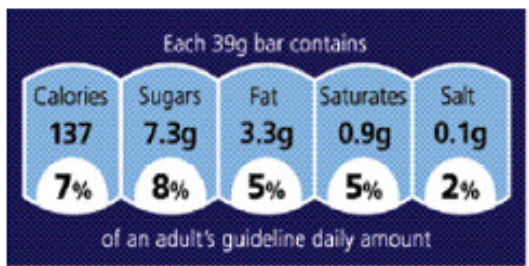

Figure 1b: Traffic Light label

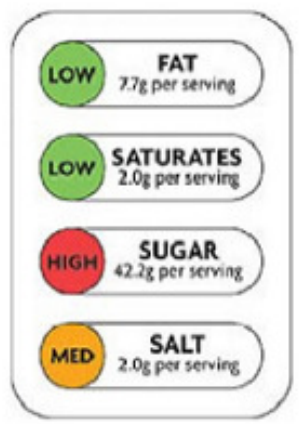

Figure 1c: Nutrition Facts label

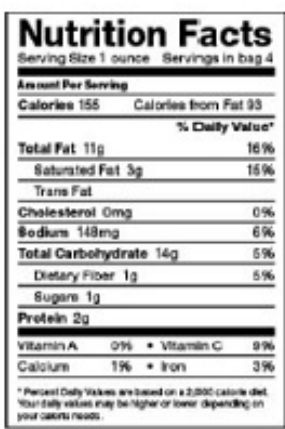

1. 'Traffic light' labels which indicate the levels of key nutrients in a portion relative to needs, as defined by the Food Standards Agency (Sacks 2009), in a high (red), medium (amber) or low (green) format (see Figure 1b).

Nutritional labelling of processed, ready-to-eat foods is now supported internationally as a means to enable consumers to make healthier choices both about what is purchased and how it is consumed (World Health Organisation 2004).

Nutritional labels take a variety of forms and for the purposes of this review three characteristics are considered necessary for a label to count as a nutritional label.

1. Types of nutrient. Information is given about one or more of the types of nutrients or energy contained in the product. These nutrients typically include those for which reductions in intake are recommended, including fats, salt and sugar. These nutrients may also include those for which increases in intake are recommended, such as vitamins or minerals. Warning labels which give information about product content that poses an immediate health threat to some people, for example 'contains peanuts' are not considered to be nutritional labels. Figure 1c shows the US nutrition facts label indicating a range of nutrients about which information may be given.

2. Amount of nutrient. Information is given indicating the amount of the different types of nutrients or energy contained within the product or a serving of the product. This information may be relative or absolute. If relative, the label will use a descriptor of the amount of the nutrient or energy contained in the product suggesting that the product is lower or higher in this nutrient than in other similar products. Thus 'low fat' or 'reduced salt' would be considered a nutritional label as 'low' and 'reduced' are descriptors of amount. Figure $1 \mathrm{~b}$ shows the use of 
relative amounts on a traffic light label. However, 'a good source of vitamin C' or 'contains whole grain' would not be considered a nutritional label as 'good' and 'contains' are not descriptors of amount. If absolute, the label will use numeric information about content, for example 'total fat - 12 grams'. Figure 1a and c show the presentation of absolute amounts.

3. Visibility. The labels can be seen when decisions about food purchasing and consumption are being made. In some cases the label will be placed directly on the front or back of packages or containers of foods purchased in food shops. In other cases the label will not appear on the product itself but will have a direct association with the specific food to which it refers. Examples include labels on a shelf on which the food is being displayed in a food shop, on the exterior of a vending machine selling snacks, on the counter from which the food is being served in a canteen, or on a restaurant menu from which food is being selected.

Additionally, labels may use formatting of the information, often termed signposting, to help individuals understand how much of the product might be eaten, and how often, as part of a healthy diet. An example of signposting is the traffic light labelling shown in Figure 1b. Any nutritional labels that do not have the characteristics specified in points 1 to 3 above will be considered as an incomplete label.

\section{How the intervention might work}

\section{Effects of nutritional labelling}

Nutritional labelling is expected to have an effect on population health through its effects on food purchasing and, ultimately, consumption. In the context of this review, healthier food purchasing and consumption are defined as:

1. increased purchasing, consumption of products and nutrients with a more healthy nutrition profile;

2. decreased purchasing, consumption of products and nutrients with a less healthy nutrition profile.

\section{Defining healthy nutrient profiles}

In the context of this review, the healthiness of a particular food product will be considered in relation to other food products tested. For example, a product that has higher levels of a nutrient, such as saturated fat, which is recommended to form a small part of dietary intake, may still be judged as healthier than a product that has an even higher level of saturated fat. To assess the absolute healthiness of products forming part of interventions included in this review would require knowledge of the full nutritional content of each product offered to study participants and more information on the context in which it was consumed relative to other components of the diet. Therefore where purchasing or consumption of more or less healthy products is the outcome of interest, the definition of a more or less healthy product will be based on the relative composition of the items tested, with reference to international dietary guidelines. Where purchasing or consumption of overall levels of specific nutrients/energy is the outcome of interest, assessments of whether labelling resulted in healthier purchasing or consumption will be based on whether the label resulted in (a) reduced purchasing/consumption of targeted nutrients/energy or (b) increases in purchasing/ consumption of targeted nutrients.

\section{Process by which nutritional labelling may have an impact on food choices and health}

The process by which nutritional labelling might be expected to lead to healthier food purchasing and consumption, resulting in better health, is shown in the logic model in Figure 2 (WK Kellogg Foundation 2004). Purchasing and consumption are influenced by the environment in which many tempting foods are available. Nutritional labelling has been proposed as an intervention that enables individuals to make healthier choices about what foods they purchase and how they consume them (Cowburn 2005; World Health Organisation 2004). Such behaviour is likely to be affected by a variety of factors. 
Figure 2. Logic model of the process by which nutritional labelling may have an impact on diets and health

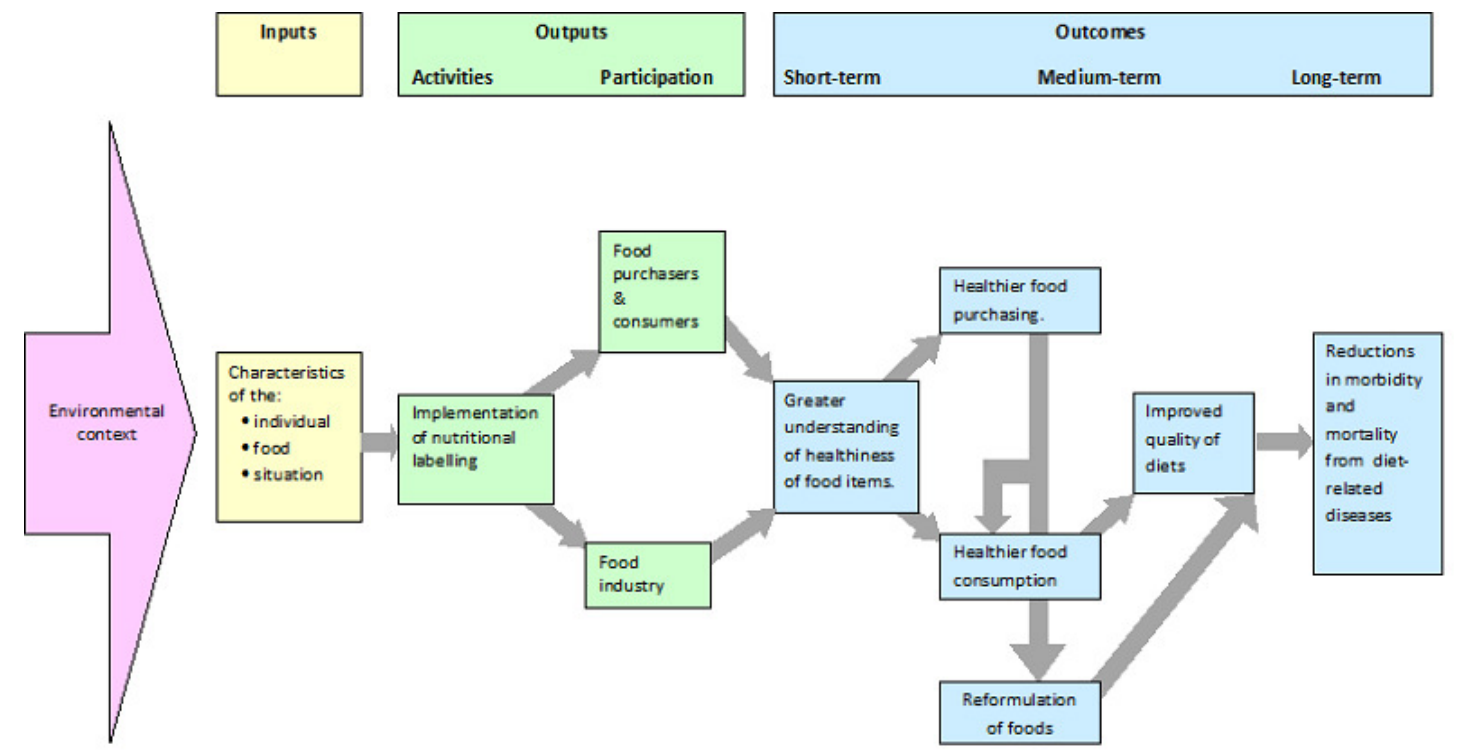

I. The label, the impact of nutritional labelling may be modified by the characteristics of the label:

1. labels presenting information about the absolute levels of nutrients or energy may have a different effect to those giving information about the relative levels. For example, stating the number of calories in a serving may have less impact on behaviour then stating 'low calorie';

2. labels including signposting to facilitate understanding of how the product fits into a healthy diet may have a different effect to those not including signposting.

II. Food, the characteristics of the labelled food may modify the effects of the label on behaviour including:

1. expectations of the taste of the food (Wansink 2004);

2. the price of the food (Horgen 2002).

III. Context, the characteristics of the situations in which the behaviour takes place, including the:

1. immediate context in which the food is purchased and consumed. For example there is evidence to indicate that whether food is purchased in a fast food or non-fast food restaurant can modify the impact of the label on purchasing (Bollinger 2010; Harnack 2008; Wisdom 2010);

2. national context in which the food is purchased or consumed. Diets vary between countries (Brownell 2006) and such differences may have an impact on the behavioural effects of labels.

IV. Individual factors, there is evidence to show that a number of factors relating to the individual may modify the effect of labels on behaviour (Campos 2011). These include:

1. dietary restraint, that is whether or not an individual is actively trying to restrict their calore intake (Aaron 1995);

2. body weight (Wansink 2006);

3. socioeconomic status (Malam 2009);

4. gender (Aaron 1995).

\section{Direct and indirect impact of labels on the individual}

The use of nutritional labels may have an impact not only on individuals who purchase or consume the food, or both, but also on the food industry in terms of encouraging the reformulation of products so that foods meet the standards to display labels indicating healthier purchases. Figure 2 indicates the possible impact of nutritional labelling on the behaviour of the food industry, directly through stimulation of reformulation of products and indirectly from changes in individual level purchasing. Understanding the impact of nutritional labelling on individual behaviour may contribute to future work exploring the impact of nutritional labelling on the food industry as, in the longer term, the response of the food industry is likely to be determined by the expected and actual behaviour of individuals in response to labels. Thus, this review focuses on the impact of nutritional labelling on individual consumer behaviour.

\section{Outcomes of nutritional labelling}

In terms of outcomes, as shown in Figure 2, in the short term there is evidence to indicate that nutritional labelling can enable pur- 
chasers to more readily assess the nutritional content of the food options available, and how they fit into a healthy diet (Feunekes 2008). This understanding is proposed to contribute to healthier food purchasing and consumption, potentially leading in the longer term to improved population health. However, it should be noted that while the logic model proposes a positive impact of nutritional labelling on purchasing and consumption, such labels may have no impact, or even a negative impact. For example, individuals may eat greater amounts of foods labelled as more healthy but these amounts may be such that they result in healthier options being consumed in unhealthily large quantities (Wansink 2006).

\section{Why it is important to do this review}

Although nutritional labelling has been widely implemented in North America, Europe and Australasia, there is no consensus as to whether it is effective in achieving healthier purchasing and consumption, nor which, if any, labels are most effective. The variety of schemes implemented may contribute to confusion. The absence of consensus on nutritional labelling policy is reflected in different recommendations about implementation that are being made internationally. For example, while the use of guideline daily amounts are being recommended in the European Union (European Commission 2011) a recent report has recommended the implementation of traffic light labels in Australia (Blewett 2011). There is thus a need for robust evidence to support decisions regarding the implementation of nutritional labelling and the development of food policy and programmes globally.

\section{Nutritional labelling and food purchasing}

There are two main areas in which studies have been conducted to ascertain the impact of nutritional labelling on food purchasing. These are purchasing in restaurants and purchasing in food shops. In terms of purchasing in restaurants, the impact of calorie (or kilojoule) labelling on restaurant menus may depend on the type of restaurant from which the food is purchased. Some research has indicated that where food is purchased from coffee shops and workbased canteens, calorie labelling increases purchasing of healthier options (Bollinger 2010; Chu 2009; Cinciripini 1984; Jensen 2009; Milich 1967) but has no effect in fast food restaurants (Elbel 2009; Harnack 2008; Wisdom 2010). The latter finding suggests that when people expect a food to be unhealthy, labelling confirms what was expected with no consequent impact on behaviour.

The impact of food labelling on food purchasing in shops can be assessed through the use of supermarket sales data. Two studies found no overall effect of 'traffic light' labels on purchases of ready meals and sandwiches (Sacks 2009; Sacks 2011). A third study found no overall effect of 'low fat', 'low calorie' or 'low trans fat' labels on the purchasing of popcorn in supermarkets, although the different labels had different effects with an increase in purchases of products labelled 'low calorie' or 'low trans fat' and a decrease in purchases of products labelled 'low fat' (Kiesel 2009). These results indicate that different labels can have different effects on behaviour and that greater clarity about these varying effects is required.

\section{Nutritional labelling, food consumption and health inequalities}

Support for nutritional labelling is predicated on the assumption that healthier food purchasing will lead to healthier consumption, specifically that nutritional labelling will enable people to eat foods in amounts appropriate to a nutritionally balanced and thus healthy diet. Even when people buy healthier options, this may not lead to healthier consumption. There is some evidence to suggest that nutritional labels may, paradoxically, serve to increase rather than decrease energy consumption through excessive consumption of products labelled as healthier options (Aaron 1995; Wansink 2006). These paradoxical effects seem greatest in those who are overweight and less restrained eaters. A further concern is that diseases associated with diets with less healthy nutrient profiles, including obesity and type 2 diabetes, are more prevalent in socially deprived groups (Foresight 2007). Current inequalities in health would be widened if nutritional labels were disproportionately effective in increasing healthy eating in those who are least socially deprived. There is evidence that nutritional labelling might have this effect as those with lower literacy from more socially deprived groups were less likely to interpret nutritional labels correctly (Malam 2009). The impact of nutritional labelling on health inequalities between low and middle income countries (LMICs) and high income countries is also a matter of concern. Given that energy dense diets are becoming cheaper and more accessible in LMICs and that in these countries rates of weight related illness are increasing (Brownell 2006; Yach 2006), it is important to ensure that nutritional labelling has beneficial effects across different countries.

The current review will identify and collate existing research evidence concerning the impact of nutritional labels on food purchasing and consumption to assess whether nutritional labelling has beneficial or adverse effects on diets, and the factors which modify these effects.

\section{O B J E C T I V E S}

The primary objective of this review is to assess whether nutritional labelling of foods in comparison to the same foods presented either without a label or with an incomplete label promotes:

1. healthier food purchasing behaviour from: a) restaurants, b) food shops, c) vending machines; or

2. healthier food consumption behaviour. 
The secondary objective of the review is to explore possible modifiers of the effect of nutritional labelling on purchasing and consumption (described in more detail under 'Types of outcome').

\section{METHODS}

\section{Criteria for considering studies for this review}

\section{Types of studies}

Studies will only be included in the review if they explore the impact of nutritional labelling on food purchasing or consumption. As the secondary objective is to explore modifiers of this effect, the same studies will be used to explore the primary and, where reported, secondary objectives. Eligible studies will be one of the following types.

1) Randomised experimental or intervention studies in which purchasing or consumption of a food product is compared between participants randomised to a control arm and one or more intervention arms in which participants see a nutritional label. This will include cluster randomised studies where particular sites, for example supermarkets, are randomised to the control or intervention groups and all participants at the particular site are included in that group. Quasi-randomised studies, where the randomisation sequence is not truly random (Reeves 2008), will be included because of the difficulty of implementing true randomisation at an aggregate, population level.

2) Interrupted time series studies in which food purchasing or consumption are compared before and after the implementation of nutritional labelling. In line with the Cochrane Effective Practice and Organisation of Care (EPOC) group recommendations, interrupted time series studies will only be included if they have a clearly defined time point at which the intervention occurred and at least three observations both pre and post-intervention (EPOC 1998). Based on Cochrane recommendations, studies that report only a simple pre and post-intervention $\mathrm{t}$-test will not be included in the review unless a valid justification for their inclusion can be made or a re-analysis of the data is possible using multiple ttests, analysis of variance or repeated measures designs (Cochrane Public Health Review Group 2010; EPOC 1998).

3) Controlled before and after studies in which food purchasing or consumption is measured before and after implementation of an intervention in non-randomised intervention and control groups. To be included in the review there must be at least two intervention sites and two control sites and characteristics of those included in the different groups should be similar. Additionally, the time that elapses between before and after measures should be comparable across groups (Ryan 2009).

Complex designs in which nutritional labelling is one of a number of the interventions implemented will be included if the effect size of the impact of the nutritional label on purchasing or consumption can be isolated.

\section{Types of participants}

Adults or children purchasing or consuming food as part of one of the included studies. Food purchases will be those bought by the individual for their personal consumption only, or for consumption by a wider group of which they are a part, for example the family of the purchaser. Food purchases will include those from any retail outlet including supermarkets and other food stores, vending machines, canteens and both fast food and non-fast food restaurants.

\section{Types of interventions}

Interventions are those that include presentation of a food product with a nutritional label, as described above, where the behavioural impact of the presentation of a label can be compared to a group in which participants see the same food product presented without a label or with an incomplete label. It should be noted that health claims will not be considered as nutritional labelling interventions in this review. Health claims are used as part of the manufacturer's marketing of a product and make a claim that the food, or a nutrient contained within it, is beneficial to general health ,for example 'healthy choice'; or a specific aspect of health, for example 'helps maintain a healthy heart'. We do acknowledge that participants' purchasing or consumption decisions may be affected by a variety of aspects of the food packaging, including health claims. However, in view of the difficulty of isolating each possible aspect of the food packages used in included studies, in this review we will focus only on the impact of nutritional labels.

\section{Types of outcome measures}

Included studies must report a behavioural outcome in terms of food purchased or consumed (see more specific parameters below).

\section{Primary outcomes}

The following are the primary outcomes for the review, when behaviourally assessed.

1. Food purchased from a supermarket or other food store.

2. Food purchased in a restaurant, canteen or other situation in which ready prepared meals are sold.

3. Food purchased from vending machines.

4. Food consumed in real world or laboratory settings.

5. Harm or unintended consequences associated with the process or outcomes of the intervention. 


\section{Food purchasing}

Food purchases from food stores, restaurants and from vending machines will be analysed separately. Healthier food purchasing will be considered to be:

1. fewer purchases of less healthy items or overall reductions in purchases of nutrients or energy targeted by the intervention for reduction;

2. more purchases of more healthy items or overall increases in purchases of nutrients, such as vitamins and minerals, targeted by the intervention for increase.

Our planned analysis of purchasing data is based on a preliminary examination of the literature. Where continuous data are provided, indicating the total amount of nutrients or energy purchased, the difference in nutrients or energy purchased between the intervention and control groups will be analysed using the mean difference. Where data about the absolute amounts of nutrients or energy consumed are not provided but data are provided about the number of participants in the control and intervention groups making a healthy choice, data will be treated as dichotomous. An initial examination of the literature suggests that it will be clear from the study report what is considered to be a healthier choice, for example the percentage of participants choosing 'low fat' or 'low calorie' options in the intervention and control groups (French 2001; Jensen 2009). Although data for different nutrients or energy will be combined in one meta-analysis for continuous outcomes and one meta-analysis for dichotomous outcomes, subgroup analyses will also be conducted to compare the impact of labelling different nutrients or energy on purchasing.

Food purchases may be assessed either at the individual or population level. At the individual level, a purchasing outcome measure will involve direct observation of what is purchased.

1. In a restaurant this observation will consist of a record of everything purchased by the individual for consumption on that visit, or a record of items targeted in the intervention purchased for consumption on that visit, for example a soft drink.

2. In a retail store the observation will consist of a record of everything purchased by the individual on that visit, or a record of items targeted in the intervention purchased on that visit, for example ready meals.

3. From a vending machine the observation will consist of a record of everything purchased by the individual on that visit, or a record of items targeted in the intervention purchased on that visit, for example a soft drink.

At a population level purchasing data will be sales data supplied by the retailer from till receipts. Such data may be presented as either sales of specific items (Sacks 2009) or as total nutrients or energy purchased (Bollinger 2010), calculated from the sales data provided. Where the purchasing is assessed in terms of the overall healthiness of the specific product labelled, it is expected that analyses presented in research reports will indicate which products are considered more or less healthy in line with the labelling given. Comparisons will be made between purchases made in a period prior to the implementation of nutritional labelling and purchases made in a comparable period following the implementation of nutritional labelling, or between similar outlets in similar locations that have and have not implemented nutritional labelling.

\section{Food consumption}

Healthier food consumption will be classified as:

1. lower consumption of less healthy foods or an overall reduction in consumption of target nutrients or energy;

2. greater consumption of more healthy foods or an overall increase in consumption of a target nutrient.

Food consumption will be assessed as an objective measure in which the amount of a snack or meal consumed will be calculated by subtracting the amount of food remaining, after consumption, from the amount of food served. This may be specified as either the:

1. amount of a food consumed, or

2. total target nutrients or energy consumed as part of a meal. Specification of both of the above is based on an initial examination of the literature. In terms of the first measure, it is expected that the food consumed will be homogeneous, for example a snack of popcorn, allowing direct comparison of the amounts consumed between study groups. In terms of the second, it is expected that the food consumed will be heterogeneous. For example, participants might be given a meal of burger and fried potatoes. Prior to the study the relevant nutritional content of each element of the meal (for example bread, meat, dressing and fried potatoes) will have been quantified. Following the meal the total target nutrient or energy consumed will be calculated by subtracting the remainder of each element of the participant's portion from the portion served. Although data for different nutrients or energy will be combined in one meta-analysis, subgroup analyses will also be conducted to compare the impact on consumption of labelling different nutrients.

\section{Secondary outcomes}

There are no secondary outcomes.

\section{Search methods for identification of studies}

The Cochrane Database of Systematic Reviews will be searched using the terms "healthy eating", "behaviour change", "health message", "obesity prevention" and "dietary intervention". The papers included in relevant reviews will be extracted.

\section{Electronic searches}

Computerised searches of the following databases will be conducted.

Health 
MEDLINE (Ovid SP) (1950 to the present)

EMBASE (Ovid SP) (1980 to the present)

CINAHL (EBSCO Host) (1982 to the present)

Cochrane Central Register of Controlled Trials (CENTRAL) (The

Cochrane Library)

Public Health

Trophi (Evidence for Policy and Practice Information (EPPI) Centre Database) (2004 to the present)

Cochrane Public Health Group Register

\section{Food}

Food Science and Technology Abstracts (FSTA)

Psychology

PsycINFO (Ovid SP) (1985 to the present)

\author{
Social science \\ Applied Social Sciences Index and Abstracts (ASSIA) from Cam- \\ bridge Scientific Abstracts (CSA) (1987 to the present) \\ Social Policy and Pratice (Ovid) (1981 to the present) \\ Sociological abstracts
}

\section{Business and marketing}

ABI Inform (Proquest) (1923 to the present)

\section{Multidisciplinary databases \\ SCOPUS (Elsevier) (1996 to the present)}

Web of Science (ISI) (Science Citation Index (1900 to the present) and Social Science Citation Index (1956 to the present))

\section{Grey literature}

Health Management Information Consortium (HMIC) (Ovid) (1983 to the present)

A draft search strategy for searching MEDLINE is shown in Appendix 1. Papers in all languages will be included. Papers identified for inclusion in the review will be used to search for further papers using both the lists of references cited by the papers as well as Web of Science cited reference searches to identify subsequent papers which cite the original study.

\section{Searching other resources}

Authors of all included studies and key researchers in the area will be contacted and asked to identify unpublished or ongoing research in the field. Additionally, the websites of key organisations in the area of health and nutrition will be searched including the following.
1. Departments of Health for England, Scotland, Wales and Northern Ireland.

2. Australian Federal and State Departments of Health.

3. Department of Health for South Africa.

4. Ministry of Health and Family Welfare for India.

5. Health Canada.

6. Food Standards Agency, UK.

7. European Commission.

8. The Rudd Centre for Food Policy and Obesity, USA.

9. Centres of Disease Control and Prevention, USA.

10. The World Health Organization.

11. National Institutes for Health Office of Disease Prevention, USA.

12. International Obesity Task Force.

\section{Data collection and analysis}

\section{Selection of studies}

The titles and abstracts of the complete list of papers identified by the literature review will be independently assessed by two review authors against the inclusion criteria. Papers identified by both review authors as not meeting all the inclusion criteria will be excluded. Papers will not be excluded on the basis of language or geographic constraints. All other papers will be subject to full-text assessment against inclusion criteria by two review authors. Any disagreements between the review authors as to whether a paper meets all the inclusion criteria will be resolved by consensus. If a consensus cannot be attained a third author will be asked to arbitrate in reaching a final decision about inclusion. Multiple papers reporting data from an individual study will be identified by comparing papers with one or more authors in common. The details of the studies presented will be compared to assess whether the papers arise from one study. Where it is not possible to ascertain this from the published papers, the authors will be contacted. Where multiple papers reports data from one study, the papers will be considered together as one report of the study. Where studies are excluded only on the basis of an incomplete label, the details of these studies will be tabulated separately.

\section{Data extraction and management}

All the results generated by the searches will be entered into Endnote XIII bibliographic software and duplicate references deleted. Multiple papers reporting results from one study will be considered as one study. A data extraction form will be developed based on the Cochrane Public Health template and modified to allow extraction of data specific to this review. The draft data extraction form will be piloted independently by two of the review authors to ensure that it enables reliable and accurate extraction of appropriate data. Two review authors will then independently extract all 
data. Where data are missing or unclear, authors will be contacted. Once all data are extracted, the first author will reconcile the two sets of data extraction forms. Where there are inconsistencies in extracted objective data, the correct data will be verified against the results reported in the paper. Where there remains uncertainty about objective data extraction, or where there are inconsistencies in extracted subjective data, the two data extractors will meet to discuss and reach a consensus as to the correct data extraction. Once data extraction is complete, one author will enter the data into Revman and a second author will check data entry.

\section{Assessment of risk of bias in included studies}

Risk of bias for randomised trials and experimental studies will be assessed using The Cochrane Collaboration tool (Higgins 2008) on the following domains.

1. Was allocation sequence adequately generated?

2. Was allocation adequately concealed?

3. Was knowledge of the allocated interventions adequately prevented during the study?

4. Were incomplete outcome data adequately addressed?

5. Are reports of the study free of suggestion of selective outcome reporting?

6. Was the study apparently free of other problems that could put it at risk of bias?

For interrupted time series studies the Cochrane Public Health Review Group Guidelines for assessing risk of bias will be implemented (Cochrane Public Health Review Group 2010), specifically the following.

1. Was the intervention independent of other changes?

2. Was the shape of the intervention effect pre-specified?

3. Was the intervention unlikely to affect data collection?

4. Was knowledge of the allocated interventions adequately prevented during the study?

5. Were incomplete outcome data adequately addressed?

6. Was the study free from selective outcome reporting?

7. Was the study apparently free of other problems that could put it at risk of bias?

To enable us to estimate risk of bias in controlled before and after studies and compare the risk of bias between the three different types of studies included in the review we will also use the Quality Assessment Tool for Quantitative Studies (EPHPP 2009). In addition, for all types of studies the risk of bias in terms of the measurement of the primary outcomes will be assessed. Low risk of bias in terms of food purchases will be considered to be where food purchases are directly observed and recorded or, at an aggregate level, sales data are monitored. In terms of food consumption, low risk of bias will be considered to be where a food portion is objectively weighed before and after consumption. Where other measures of the primary outcomes are used, or where the method by which this measurement was made is not stated, the study will be considered to be at high or unclear risk of bias. Where necessary, study authors will be contacted to provide further information. The risk of bias of each study on each indicator will be shown in a risk of bias table. Based on these domains, a specific table will be included in the data extraction form to allow risk of bias to be recorded.

\section{Measures of treatment effect}

Dichotomous and continuous data will be combined separately and appropriate effect sizes calculated. It is expected that food purchase data will be either dichotomous, indicating that a more or less healthy choice was made, or continuous in terms of the total amount of nutrient or energy purchased. Food purchases from supermarkets and other food stores will be analysed separately to those made in restaurants. It is expected that food consumption will be assessed as a continuous, objective measure of the total nutrients or energy consumed. Dichotomous data will be analysed using odds ratios and continuous data will be analysed using mean differences between groups. The combined effect sizes will be calculated with $95 \%$ confidence intervals using a random-effects model to allow for the possibility that population effect sizes may vary between studies.

\section{Process measures}

Measures relating to the process of implementing the intervention,including any data on cost of the implementing the intervention included in the studies, will be extracted and listed in the 'Characteristics of included studies' and summarised in the 'Results' section.

\section{Unit of analysis issues}

In terms of cluster randomised trials, where the analyses presented in the primary paper have taken account of the design, the studies will be considered at lower risk of bias for the purposes of the sensitivity analyses.

\section{Dealing with missing data}

Where data are missing due to participant dropout we will conduct both available case analyses and intention-to-treat (ITT) analyses. A conservative approach will be taken in the ITT analysis for dichotomous outcomes whereby it will be assumed that participants with missing data choose the least healthy option. Where ITT analyses are not possible due to missing data or continuous data, we will analyse outcomes as reported because of the problematic nature of imputation. We will also report on levels of dropout in the intervention and comparison groups as an indicator of 'acceptability' of the intervention, and the likelihood of bias due to attrition. 


\section{Assessment of heterogeneity}

Evidence of the extent of heterogeneity will be assessed by visually examining the extent to which confidence intervals overlap. Additionally the $\mathrm{I}^{2}$ statistic, automatically calculated by the Revman software, will be reported and an interpretation of the levels of heterogeneity that are present will be made based on the recommendations of Deeks 2008.

\section{Assessment of reporting biases}

Possible reporting bias will be assessed using funnel plots exploring the relationship between effect size and study size. These will be visually examined for symmetry, with greater symmetry indicating a lower risk of reporting bias.

\section{Data synthesis}

We will describe and summarise the findings of the included studies. Where there are enough studies that are sufficiently homogeneous in terms of the intervention, methods and outcomes, we will conduct meta-analyses to assess the combined effects of the intervention on behaviour. Meta-analyses will only be carried out on results of randomised controlled trials (RCTs), cluster RCTs and quasi-RCTs. Because of the increased risk of bias, data from controlled before and after studies and interrupted time series studies will be tabulated in a form additional to the meta-analyses, summarised in a narrative synthesis and included in the discussion. We will only include studies considered to be at lower risk of bias in the meta-analysis. It has been recommended that meta-analyses should be restricted to studies considered to be at lower risk of bias (Higgins 2008). However, including only studies that are considered to be at low risk of bias on all indicators is likely to leave very few studies for inclusion in the meta-analysis. Thus, based on the risk of bias table, an assessment will be made of the extent to which each identified risk for each study may have affected the results. Where there is considered to be a high risk that the results reported in a specific paper are biased, these data will be excluded from the meta-analysis. Outcome data reported in these studies will be extracted and presented in tables describing and summarising the results of each study. The implications of these results will then be explored in the discussion.

\section{Analysis of primary outcomes}

Separate meta-analyses will be conducted for the effects of nutritional labelling on:

1. food purchases from restaurants;

2. food purchases from supermarkets and other food shops;

3. food purchases from vending machines;

4. food consumption.

Because there is likely to be some degree of heterogeneity, a random-effects model will be used to obtain the combined effect size with $95 \%$ confidence interval. For dichotomous outcomes the combined odds ratio of purchasing or consuming a more healthy rather than a less healthy food option will be assessed. We expect continuous outcomes to be objectively assessed in terms of weight of food consumed or energy contained in the food. The expected homogeneity of measurement means that these data can be combined using the mean difference. Meta-analyses will be conducted using Revman 5. For dichotomous data, Mantel-Haenszel methods will be used, which are the default methods in Revman. For continuous data the inverse variance approach will be used.

\section{Subgroup analysis and investigation of heterogeneity}

Subgroup analyses will be used to address two different issues: the exploration of effect modifiers and the investigation of heterogeneity. Given the increased Type I error rate with multiple comparisons, should there be sufficient data to explore more than two effect modifiers we will adjust statistical significance levels accordingly.

\section{The exploration of effect modifiers}

Where reported, modifiers of the impact of nutritional labelling on purchasing and consumption will be explored. The specific modifiers to be explored are described in the section above on 'How the intervention might work' and in this section, below. It is expected that the following effect modifiers will be assessed using measures allowing specification of the same dichotomous categories across studies:

1. body weight: overweight (> BMI 25) or not overweight (< BMI 25);

2. dietary restraint, restrained eater (is dieting) or unrestrained eater (is not dieting);

3. gender, male or female;

4. label amount formats, relative amounts or absolute amounts of the nutrient or energy;

5. label signposting, signposting present or absent;

6. the national context in which food is purchased or consumed. Initial examination of the literature indicates that a large proportion of the current research originates in the United States of America (US). Thus effects of nutritional labelling assessed in the United States will be compared with effects assessed in other countries. If there is sufficient variation in the country of study, comparisons between countries will be made. The following effect modifiers may be measured on a variety of scales, making it difficult to specify the levels of interest a priori. Categorisations applied to the outcomes as assessed in each individual study will be used as a basis for specifying dichotomous categories for each of these outcomes. An assessment will be made of whether the categories specified are sufficiently similar between studies to allow data to be combined. Where there is uncertainty as to whether the levels specified in the different studies represent similar levels of the construct across studies, the data will not be used in the exploration of effect modification. 
1. Socioeconomic status: more socially deprived or less socially deprived.

2. Expectations of the taste the food: tastes poor or tastes good.

3. The price of the food, more expensive or less expensive.

4. The immediate context in which food is:

i) purchased, food purchased in a fast food restaurant or non-fast food restaurant;

ii) consumed, food consumed in a natural setting or a laboratory setting.

For each of the primary outcomes (food purchasing from restaurants, food shops and vending machines; and food consumption) the modifying effects of any of the 10 possible modifiers above, for which sufficient data are available, will be assessed. The only exceptions to this procedure is in regards to the immediate context in which food is: (a) purchased or (b) consumed. In terms of purchasing, the subgroup analyses will compare the impact of nutritional labelling on food purchased from a fast food restaurant to that from a non-fast food restaurant. For this analysis data on food purchased from food stores and vending machines will be excluded. In terms of consumption, subgroup analyses will compare the impact of nutritional labelling on consumption in a natural setting with that in a laboratory setting. The exploration of these effect modifiers will be conducted using the procedure outlined below.

Modifiers of the impact of nutritional labels are of two kinds:

1. study level modifiers or between study effects, for example different effects of the intervention in studies from different countries;

2. within study moderators, for example different effects of the intervention in different groups such as men and women or obese versus non-obese.

The precise methods used to explore these two types of effect will depend on whether the primary outcome being assessed is continuous or dichotomous.

1. Study level moderators with continuous outcomes, for example energy consumption in studies from the US and from the rest of the world: the difference in mean energy consumption between the intervention and control group for each study will be ascertained and the difference in pooled mean difference between studies from the US and the rest of the world will be ascertained using subgroup analysis.

2. Within study moderators with continuous outcomes, for example energy consumption among men and women: in each study, difference in mean energy consumption between the intervention and control among men and among women will be calculated. Pooled mean difference between men and women will be ascertained using subgroup analysis.

3. Study level moderators with dichotomous outcomes, for example the choice to purchase a more healthy item or not in studies from the US and studies from the rest of the world: the pooled odds ratios for studies from the US and from the rest of the world will be calculated. The pooled odds ratio for studies from the US will be divided by the pooled odds ratio for studies from the rest of the world to give a relative odds ratio with confidence interval.

4. Within study moderators with dichotomous outcomes, for example the choice to purchase a more healthy item in men and women: for each study the odds ratio for men and the odds ratio for women will be calculated and the odds ratio for men will be divided by the odds ratio for women to give the relative odds ratio. The pooled relative odds ratio can then be ascertained.

\section{Investigation of heterogeneity}

There are three possible sources of heterogeneity that will be explored using subgroup analysis.

1. The impact of the positioning of the label, comparing those that appear on the food package with those appearing in another location, such as on a supermarket shelf.

2. The impact of the information given on the label. First, labels giving information about a range of nutrients will be compared with those giving information about one nutrient. Second, as labels most frequently give calorie information, we will compare the impact of labels giving information about calorie content with labels giving information about other nutrients.

3. The impact of the definitions of healthy purchasing and healthy consumption used in this review. More healthy purchasing is considered to be decreased purchasing of less healthy foods or increased purchasing of more healthy foods but it is possible that these are two separate behaviours. Subgroup analysis will be used to identify whether they are separate behaviours and this a source of heterogeneity. Similarly possible heterogeneity as a consequence of defining more healthy consumption as either decreased consumption of less-healthy foods or increased consumption of more-healthy foods will be investigated.

\section{Sensitivity analysis}

Sensitivity analyses will be conducted to explore the impact of missing data comparing results from available-case and ITT analysis. Sensitivity analyses will also be used to assess the effects of nutritional labelling on behaviour across studies at both high and low risk of bias, specifically the meta-analyses will be re-run including all studies regardless of their risk of bias. Additionally, the impact of the definition of nutritional labels used in this review will be explored. The meta-analyses will be re-run including the studies excluded from the main analyses due to the presentation of an incomplete label rather than a complete label (as described in the 'Description of the intervention').

\section{ACKNOWLEDGEMENTS}


The development of this review protocol has been informed by members of a Review Advisory Group.

\section{R E F E R E N C S}

\section{Additional references}

\section{Aaron 1995}

Aaron JI, Evans RE, Mela DJ. Paradoxical effect of a nutrition labelling scheme in a student cafeteria. Nutrition Research 1995;15:1251-61.

\section{Blewett 2011}

Blewett N, Goddard N, Pettigrew S, Reynolds C, Yeatman H. Labelling Logic: Review of Food Labelling Law and Policy. http://www.foodlabellingreview.gov.au/ internet/foodlabelling/publishing.nsf/content/labellinglogicGovernment Report. Canberra: Commonwealth of Australia, 2011.

\section{Bollinger 2010}

Bollinger B, Leslie P, Sorensen A. Calorie posting in chain restaurants. National Bureau for Economic Research Working Paper Series. National Bureau for Economic Research, 2010.

\section{Brownell 2006}

Brownell K, Yach D. Lessons from a small country about the global obesity crisis. Globilization and Health 2006;2(1):11.

\section{Cabinet Office 2008}

Cabinet Office. Food Matters: Towards a Strategy for the 21st Century. http://www.cabinetoffice.gov.uk/media/ cabinetoffice/strategy/assets/food/food matters 1.pdf. Cabinet Office, 2008.

\section{Campos 2011}

Campos S, Doxey J, Hammond D. Nutrition labels on pre-packaged foods: a systematic review. Public Health Nutrition 2011;FirstView:1-11.

Chu 2009

Chu YH, Frongillo EA, Jones SJ, Kaye GL. Improving patrons' meal selections through the use of point-ofselection nutrition labels. American Journal of Public Health 2009;99:2001-5.

\section{Cinciripini 1984}

Cinciripini PM. Changing food selections in a public cafeteria: An applied behavior analysis. Behavior Modification 1984;8:520-39.

Cochrane Public Health Review Group 2010 Cochrane Public Health Group. Guideline for Developing a Cochrane Protocol. http:// ph.cochrane.org/sites/ph.cochrane.org/files/uploads/ HPPH 'systematic' review 'handbook.pdf 2010.

\section{Cowburn 2005}

Cowburn G, Stockley L. Consumer understanding and use of nutrition labelling: a systematic review. Public Health Nutrition 2005;8(1):21-8.
Deeks 2008

Deeks JJ, Higgins JPT, Altman DG. Chapter 9: Analysing data and undertaking meta-analyses. In: Higgins JPT,

Green S editor(s). Cochrane Handbook for Systematic Reviews of Interventions. The Cochrane Collaboration, 2008.

Elbel 2009

Elbel B, Kersh R, Brescoll VL, Dixon LB. Calorie labeling and food choices: a first look at the effects on lowincome people in New York City. Health Affairs 2009;28: w1110-21.

\section{EPHPP 2009}

Effective Public Health Practice Project. Quality Assessment Tool for Quantitative Studies. http://www.ephpp.ca/ tools.html 2009.

\section{EPOC 1998}

Cochrane Effective Practice and Organisation of Care Group. Including Interrupted Time Series Designs in a EPOC Review. http://epoc.cochrane.org/sites/ epoc.cochrane.org/files/uploads/inttime.pdf 1998.

European Commission 2011

European Commission. Provision of Food Information to Consumers - Proposed Legislation. http:// ec.europa.eu/food/food/labellingnutrition/foodlabelling/ proposed legislation 'en.htm. European Community, 2011.

\section{Feunekes 2008}

Feunekes GI, Gortemaker IA, Willems AA, Lion R, Van den Kommer M. Front-of-pack nutrition labelling: Testing effectiveness of different nutrition labelling formats frontof-pack in four European countries. Appetite 2008;50(1): 57-70.

Finkelstein 2003

Finkelstein E, Fiebelkorn I, Wang G. National medical spending attributable to overweight and obesity: how much, and who's paying. Health Affairs - Web Exclusive 2003;W3:219-26.

Foresight 2007

Foresight. Tackling Obesities: Future Choices. http:// www.bis.gov.uk/assets/bispartners/foresight/docs/obesity/ 17.pdf. London: Government Office for Science, 2007.

\section{French 2001}

French S, Jeffrey R, Story M, Breitlow K, Baxter J, Hannan P, Snyder P. Pricing and promotion effects on low fat vending snack purchases. American Journal of Public Health 2001;91(1):112-7.

\section{Harnack 2008}

Harnack LJ, French SA, Oakes JM, Story MT, Jeffery RW, Rydell SA. Effects of calorie labeling and value size pricing on fast food meal choices: Results from an experimental 
trial. International Journal of Behavioral Nutrition and Physical Activity 2008; Vol. 5:63.

\section{Higgins 2008}

Higgins JPT, Altman DG. Chapter 8: Assessing risk of bias in included studies. In: Higgins JPT, Green S editor(s). Cochrane Handbook for Systematic Reviews of Interventions. The Cochrane Collaboration, 2008.

\section{Horgen 2002}

Horgen KB, Brownell KD. Comparison of price change and health message interventions in promoting healthy food choices. Health Psychology 2002;21:505-12.

\section{Jensen 2009}

Jensen C, Webb K, Mandel S, Hudes M, Crawford P. Evaluation of the pilot menu labelling initiative in Kaiser Permanente cafeterias 2008. http://ucanr.org/sites/news/ files/4368.pdf. Berkeley: Dr Robert C. and Veronica Atkins Center for Weight and Health, University of California, 2009.

\section{Kiesel 2009}

Kiesel K, Villas-Boas S. Can information costs confuse consumer choice? Nutritional labels in a supermarket experiment. http://escholarship.org/uc/item/6st6d0rr 2009.

\section{Malam 2009}

Malam S, Clegg S, Kiwan S, McGinigal S, BMRB Social Research. Comprehension and use of UK nutritional signpost labelling schemes.. http://www.food.gov.uk/ multimedia/pdfs/pmpreport.pdf 2009.

\section{Milich 1967}

Milich R, Anderson J, Mills M. Effects of visual presentation of caloric values on food buying by normal and obese persons. Perceptual and Motor Skills 1967;42:155-62.

\section{Ogden 2007}

Ogden J, Karim L, Choudry A, Brown K. Understanding successful behaviour change: the role of intentions, attitudes to the target and motivations and the example of diet. Health Education Research 2007;22(3):397-405.

\section{Reeves 2008}

Reeves B, Deeks J, Higgins J, Wells G. Chapter 13: Including non randomised studies. In: Higgins JPT, Green S editor(s). Cochrane Handbook for Systematic Reviews of interventions. The Cochrane Collaboration, 2008.

\section{Ryan 2009}

Ryan R, Hill S, Broclain D, Horey D, Oliver S, Prictor M. Study Design Guide. Cochrane Consumers and Communication Group July 2009.

\section{Sacks 2009}

Sacks G, Rayner M, Swinburn B. Impact of front-of-pack 'traffic-light' nutrition labelling on consumer food purchases in the UK. Health Promotion International 2009;24:344-52.

\section{Sacks 2011}

Sacks G, Tikellis K, Millar L, Swinburn B. Impact of 'trafficlight' nutrition information on online food purchases in Australia. Australian and New Zealand Journal of Public Health 2011;35(2):122-6.

\section{Sutton 1998}

Sutton S. Predicting and explaining intentions and behavior: How well are we doing?. Journal of Applied Social Psychology 1998;28(15):1317-38.

\section{Wansink 2004}

Wansink B, van Ittersum K, Painter JE. How diet and health labels influence taste and satiation. Journal of Food Science 2004;69(9):S340-6.

\section{Wansink 2006}

Wansink B, Chandon P. Can "Low-Fat" nutrition labels lead to obesity?. Journal of Marketing Research 2006;43:605-17.

\section{Wisdom 2010}

Wisdom J, Downs J, Loewenstein G. Promoting healthy choices: Information vs. convenience. American Economic Journal: Applied Economics. 2010;2(2):164-78.

\section{WK Kellogg Foundation 2004}

WK Kellogg Foundation. Logic Model Development Guide. http://www.wkkf.org/knowledge-center/resources/ 2010/Logic-Model-Development-Guide.aspx. Michigan: WK Kellogg Foundation, 2004.

\section{World Health Organisation 2004}

World Health Organization. Global Strategy, on Diet, Physical Activity and Health. http://www.who.int/ dietphysicalactivity/en/. World Health Organisation, 2004.

\section{Yach 2006}

Yach D, Stuckler D, Brownell K. Epidemiological and economic consequences of the global epidemics of obesity and diabetes. Nature Medicine 2006;12(1):62-66.

* Indicates the major publication for the study 
A P P E N D I CES

\section{Appendix I. MEDLINE search strategy}

1. exp Food packaging/ and (label\$ or content $\$$ sign $\$$ or symbol $\$$ or ticket\$ or sticker\$ or diet\$ or health\$ or calori $\$$ or nutritio\$ or guideline daily amount $\$$ or recommended daily amount $\$$ or nutrient reference value\$ or nutrient daily value\$).ti,ab

2. food pack\$.ab,ti.

3. exp Product labelling/ and (food $\$$ or fat $\$$ or sugar $\$$ or salt or diet $\$$ or health $\$$ or calori $\$$ or nutritio $\$$ or guideline daily amount $\$$ or recommended daily amount $\$$ or nutrient reference value $\$$ or nutrient daily value $\$$ or snack\$ or eat\$).ti,ab

4. exp Food Labeling/

5. ( (Nutritio $\$$ or Nutrient $\$$ ) adj5 (label\$ or content $\$$ sign $\$$ or symbol\$ or ticket\$ or sticker\$)).ab,ti

6. (nutrition $\$$ information or nutrient $\$$ information).ti,ab

7. (Food $\$$ label\$ or food $\$$ content $\$$ label\$ or food $\$$ content $\$$ sign $\$$ or food $\$$ content symbol\$ or food $\$$ content $\$ \operatorname{tag} \$$ or food $\$$ content\$ ticket\$ or food $\$$ content\$ sticker\$).ab,ti

8. traffic light $\$ . a b, t i$.

9. (guideline daily amount\$ or nutrient reference value $\$$ or nutrient daily value $\$$ ).ab,ti

10. (recommended dietary allowance $\$$ adj5 (label\$ or content $\$$ sign $\$$ or symbol $\$$ or information or ticket $\$$ or sticker $\$)$ ).ab,ti

11. Healthy choice.ab,ti.

12. (Calorific or calorie\$ or caloric) and (label\$ or content $\$$ sign $\$$ or symbol\$ or ticket\$ or sticker\$)).ab,ti

13. ((Calorific or calorie $\$$ or caloric) adj information).ab,ti

14. (fat adj5 (label\$ or content $\$$ sign $\$$ or symbol $\$$ or tag $\$$ or ticket $\$$ or sticker\$)).ab,ti

15. (salt adj5 (label\$ or content $\$$ sign $\$$ or symbol $\$$ or tag $\$$ or ticket $\$$ or sticker\$)).ab,ti

16. (sugar adj5 (label $\$$ or content $\$$ sign $\$$ or symbol $\$$ or tag $\$$ or ticket $\$$ or sticker\$)).ab,ti

17. (menu and (label\$ or content $\$$ sign $\$$ or symbol $\$$ or tag $\$$ or ticket $\$$ or sticker\$)).ab,ti

18. (menu and (nutritional content $\$$ or nutritional information or traffic light or guideline daily amount or GDA or healthy choice or calorie or fat or sugar)).ab,ti

19. (Label\$ adj2 (legislation $\$$ or regulation $\$$ or policies or policy)

).ti,ab

20. or/1-19

21. exp Food Preferences/

22. $\exp$ Food Habits/

23. exp Feeding Behavior/

24. exp Eating/

25. $\exp$ Diet/

26. $\exp$ Choice Behavior/

Nutritional labelling for promoting healthier food purchasing and consumption (Protocol)

Copyright $\Subset 201$ I The Cochrane Collaboration. Published by John Wiley \& Sons, Ltd. 
27. (intak $\$$ or consume or consumes or consumption or consumed or eat $\$$ or diet $\$)$.ti,ab

28. (food adj5 (preference\$ or habit\$ or behavio?r\$ or choice\$ or decision $\$$ or decid $\$$ or inclin $\$$ or lik $\$$ or choos $\$$ or select $\$$ or pick\$)).ab,ti

29. or $/ 21-28$

30. exp Restaurants/

31. (purchas $\$$ or buy $\$$ or sale $\$$ or vend $\$$ or sell $\$$ ).ab,ti.

32. (shop\$ or store $\$$ or supermarket $\$$ or market $\$$ or outlet $\$$ or retailer $\$$ or point of purchase).ab,ti

33. (restaurant $\$$ or cafe $\$$ or bar $\$$ or canteen $\$$ or cafeteria $\$$ or dinner hall $\$$ or dining area $\$$ or dining room $\$$ or refector $\$$ or eatery or mess or buffet or bistro\$ or eating place\$).ab,ti

34. or $/ 30-33$

35. 20 and ( 29 or 34$)$

\section{H I S T O R Y}

Protocol first published: Issue 9, 2011

\section{CONTRIBUTIONSOFAUTHORS}

Draft the protocol background and selection criteria: RAC, GJH, SAJ, TMM

Draft the protocol methods section: RAC, GJH, SAJ, TMM

Updating the review: RAC

\section{DECLARATIONS OF INTEREST}

Susan Jebb is Chair of the Public Health Responsibility Deal Food Network. She receives no external funding for research or advisory work relating directly to labelling.

\section{SOURCES OF SUPPORT}

\section{Internal sources}

- King's College London, UK.

Provides support and resources for two authors (RAC, TMM)

- University of Cambridge, UK.

Provides support for three authors (GJH, TMM, SAJ) 


\section{External sources}

- National Institutes for Health Research, UK.

Postdoctoral Research Fellowship (RAC)

- Coca-Cola, Tanita, Sanofi Aventis, WeightWatchers, Food Standards Agency, WRCF, EU, Not specified.

Receipt of research grants in last 5 years (SAJ)

- Rosemary Conley Diet and Fitness, UK.

Fee for nutrition articles in magazine and for occasional lectures to franchisees (SAJ) 